\title{
Down syndrome Disorder Diagnosis in Children Using Analysis of Voice in Signal Processing
}

\author{
Dickson, Makasda Solomon and Kabari, Ledisi G \\ Department of Computer Science, Ignatuius Ajuru University of Education, Port Harcourt, Nigeria
}

\begin{abstract}
Down syndrome is one of the most leading causes of intellectual disability and millions of these patients face various health issues including learning and memory, congenital heart diseases (CHD), Alzheimer's diseases (AD), leukemia, cancers and Hirschprung disease (HD).

The incidence of trisomy is influenced by maternal age and differs in population (between 1 in 319 and 1 in 1000 live births) [1]. DS has high genetic complexity and phenotype variability. Trisomic fetuses are at elevated risk of miscarriages and DS people have increased incidence of developing several medical conditions $[2,3]$. Recent advancement in medical treatment with social support has increased the life expectancy for DS population. In developed countries, the average life span for DS population is 55 years [4].
\end{abstract}

\section{INTRODUCTION}

$\mathrm{D}$ own syndrome is a relatively common chromosomal disorder. Most children with Down syndrome have mild to moderate mental impairment. About half have heart defects or other medical problems such as hearing and vision problems. [5] Prenatal testing may be able to recognize Down syndrome before the baby is born. Your doctor can help to arrange for appropriate medical care for your child with Down syndrome, along with services and support for your family. [6]

Down syndrome is a genetic disease caused by a chromosomal abnormality. The child usually has a certain appearance that is common to children with Down syndrome.

These children also have differing degrees of mental deficiency, heart disease, and other problems. Down syndrome usually results from an extra copy of chromosome 21 , although other chromosomal abnormalities are possible. It occurs in about 1 of every 600 to 800 liveborn babies. [7] Your child will have screening tests to look for medical problems associated with Down syndrome. Visits to some medical specialists may be recommended as well. We can help you access appropriate medical care for your child as well as support for your family, including early intervention and special education services.

In over $90 \%$ of children with Down syndrome, the disease results from the presence of an extra chromosome. Normally, people have 46 chromosomes 23 from each parent. Most children with Down syndrome have 47 chromosomes, with an extra copy of chromosome 21; that's why this condition is also called "trisomy 21." A small number of children have the right number of chromosomes, but an extra piece of chromosome 21 is attached to one of the other chromosomes.[8] This is called a translocation, and affected children have the same problems as those with typical Down syndrome. In $1 \%$ to $2 \%$ of children with Down syndrome, some cells contain the normal 46 chromosomes, while others contain 47 chromosomes. This is called mosaicism. These children have fewer problems than those with other chromosomal abnormalities.

Digital signal processing technology, as an important product of the information age, exists in all aspects of the communication field and is an indispensable technology in the communication field. In recent years, China has increased its support for chips. Therefore, it has promoted the development and promotion of DSP chips, making it a control chip for many mainstream software communication products.[9] In the field of communications, the equipment that applies digital signal processing mainly includes systems such as telephone communications, video voice, and voice signals, which effectively promote information exchange and information sharing [10].

The paper is intended to analyzer and compared voice of child with Down syndrome and that of normal child. This can be achieve by obtaining and analyzing the voice data set of the child with down syndrome and of the normal child using MATLAB and compare normal child's voice and down syndrome child's voice using time-domain signal, frequency spectrum view of signals and spectrogram signal view in MATLAB.

The field of signal processing practice is constrained with some major challenges ranging from technology age, chips, sensors, understanding of concepts practice policy and financial factors. Digital signal processing technology, as an important product of the information age, exists in all aspects of the communication field and is an indispensable technology in the communication field. In recent years, China has increased its support for chips. Therefore, it has promoted the development and promotion of DSP chips, making it a control chip for many mainstream software communication products. In the field of communications, the equipment that applies digital signal processing mainly includes systems such as telephone communications, video voice, and voice signals, which effectively promote information exchange and information sharing. In the field of communication, digital signal processing technology is mainly applied to voice compression coding and software radio of course; there are 
still problems such as signal quality and signal transmission speed that need to be resolved.

The paper starts by introduction $t$ the research and the idea behind it. It then provides related works in speech processing and speech signal processing with interest on children with down syndrome. The way the research was carried out then followed and the results and analysis was given and finally conclusion.

\section{RELATED WORKS}

[11] Conducted a study on Down syndrome trisomy 21. After the baby is born, a simple chromosome test can confirm whether or not Down syndrome is present. Women over 35 and other pregnant women at high risk of having a Down syndrome child can undergo prenatal (before birth) testing. This may include procedures to obtain samples of the amniotic fluid in the womb (called amniocentesis) or the placenta (called chorionic villus sampling). New strategies to detect Down syndrome before birth are now being used. A combination of blood tests and ultrasound scans is performed during the first and second trimesters of pregnancy. The results help to determine if amniocentesis or other additional tests are needed. If not, the mother can avoid the risks of these tests. Similarly [12] carried out a study on Down syndrome: an insight of the disease Down syndrome (DS) is one of the commonest disorders with huge medical and social cost. DS is associated with number of phenotypes including congenital heart defects, leukemia, Alzeihmer's disease, Hirschsprung disease etc. DS individuals are affected by these phenotypes to a variable extent thus understanding the cause of this variation is a key challenge. In the present review article, we emphasize an overview of DS; DS associated phenotypes diagnosis and management of the disease. The genes or miRNA involved in Down syndrome associated Alzheimer's disease, congenital heart defects (AVSD), leukemia including AMKL and ALL, hypertension and Hirschprung disease are discussed in this article. Moreover, we have also reviewed various prenatal diagnostic methods from karyotyping to rapid molecular methods - MLPA, FISH, QF-PCR, PSQ, NGS and noninvasive prenatal diagnosis in detail.

\section{A. Speech Processing}

On average, language and communication characteristics of individuals with Down syndrome (the most common genetic cause of intellectual disability) follow a consistent profile. Despite considerable individual variability, receptive language is typically stronger than expressive language, with particular challenges in phonology and syntax. hearing, oral-motor, cognitive, social, and prelinguistic and early nonverbal communication skills of individuals with Down syndrome. Hearing loss can affect one or both ears and range from mild to profound [13]

Speech production of individuals with Down syndrome may be related to differences in oral structure and function[14]. Structural differences include a small oral cavity with a relatively large tongue and a narrow, high arched palate. Missing, poorly differentiated, or additional muscles characterize facial structures, and differences in nerve innervation have been found as well[14].

Expressive language skills present particular challenges and generally are more impaired than receptive skills in young individuals with Down syndrome[15].

A discrepancy between perceptual judgments of pitch level and acoustic measures has been noted[16], which may mean that perception of low vocal pitch is influenced by factors other than the actual frequency of vocal fold vibration. It may help to resolve this discrepancy by examining a range of acoustic and perceptual factors associated with voice production in individuals with DS, taking into account a developmental perspective that covers the period from infancy to adulthood.

Vocal quality has been studied with both perceptual and acoustic methods. The perceptual studies of voice in DS note especially breathiness and roughness. There is no complete agreement in published works, however acoustic studies report increased frequency perturbations (e.g., higher values of jitter), amplitude perturbations (e.g., higher values of shimmer) and increased noise in phonation (e.g., reduced signal-to-noise ratio, $\mathrm{S} / \mathrm{N}$ ). Discrepant results also have been reported for spectral tilt[16]. The variability in results among studies may be due in part to differences in participant samples, speaking task differences, language differences, or differences in the algorithms or equipment used to calculate the acoustic values. No single acoustic correlate of voice quality in DS consistently emerges in the published literature, nor is it clear if a particular voice quality persists in individuals with DS across various speaking tasks and if voice quality in DS changes with development. Despite frequent comments in the clinical literature on voice quality differences in DS, there has not been a satisfactory convergence on perceptual features or on acoustic correlates of voice quality.

\section{B. Speech Signal Processing}

Automatic speech recognition by machine is one of the most effective methods for man-machine communication. Because speech waveform is nonlinear and time-variant, speech recognition requires significant amount of intelligence and fault tolerance in the pattern recognition algorithms. Isolated word recognition through the use of Digital Signal Processing (DSP) and the use of MATLAB was conducted, VHSIC Hardware Description Language (VHDL) was used to extract the features of the speech signals[17].

Speech recognition systems consider that the speech signal is a realization of some message encoded as a sequence of one or more symbols. The essential goal is to decode" this message and then convert it either into writing or into commands to be processed. Speech recognition systems consider that the speech signal is a realization of some message encoded as a sequence of one or more symbols. The 
essential goal is to "decode" this message and then convert it either into writing or into commands to be processed.

Speech recognition systems can be divided into distinct classes by describing what types of utterances they can recognize. These classes can be identified as Connected Words, Continuous Speech, Spontaneous Speech, Isolated Words and Isolated word.

The speech of people with Down syndrome (DS) shows prosodic features which are distinct from those observed in the oral productions of typically developing (TD) speakers. Although a different prosodic realization does not necessarily imply wrong expression of prosodic functions, a typical expression may hinder communication skills. The focus of the work was to ascertain whether this can be the case in individuals with DS. To do so, they analyzed the acoustic features that better characterize the utterances of speakers with DS when expressing prosodic functions related to emotion, turn-end and phrasal chunking, comparing them with those used by TD speakers. An oral corpus of speech utterances has been recorded using the PEPS-C prosodic competence evaluation tool. They used automatic classifiers to prove that the prosodic features that better predict prosodic functions in TD speakers are less informative in speakers with DS[18].

\section{METHODOLOGY}

The voice analysis was conducted by comparison voice of a normal child and a child with Down syndrome based on: age, frequency, pulse, amplitude, harmonic nature of the voice and pitch. In this study, the signal analyzer application was adopted in MATLAB. The Signal Analyzer application is an interactive tool for time-domain, frequency-domain and timefrequency-domain visualization, preprocessing, measurement, analysis, and comparison of signal.

\section{RESULTS AND DISCUSSION}

The figure 1-3, shows the results analysis of child with Down syndrome and of the normal child with a frequency sample of $44100 \mathrm{~Hz}$ and $48000 \mathrm{~Hz}$ respectively. The voice results analysis of child with Down syndrome is shown in figure 1 while that of normal child in figure 2. Figure 3 shows the combination Down syndrome child and normal child signal in time-domain and frequency view.

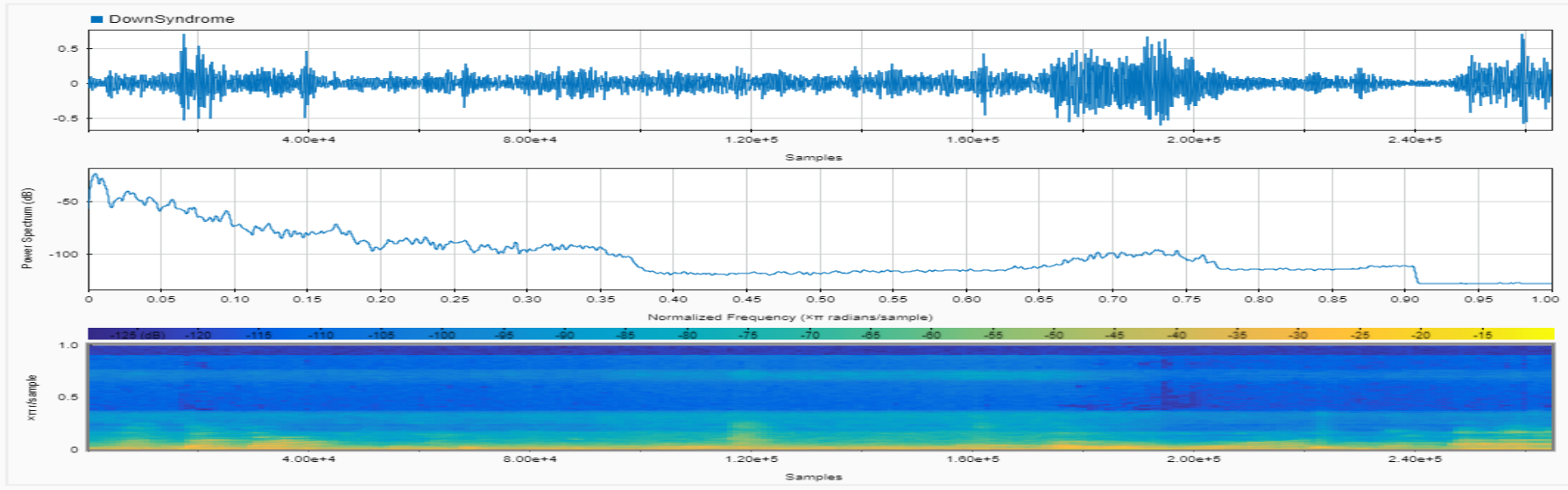

Figure 1: Voice signal of Down syndrome child on time-domain, Spectrum/Frequency, and spectrogram view

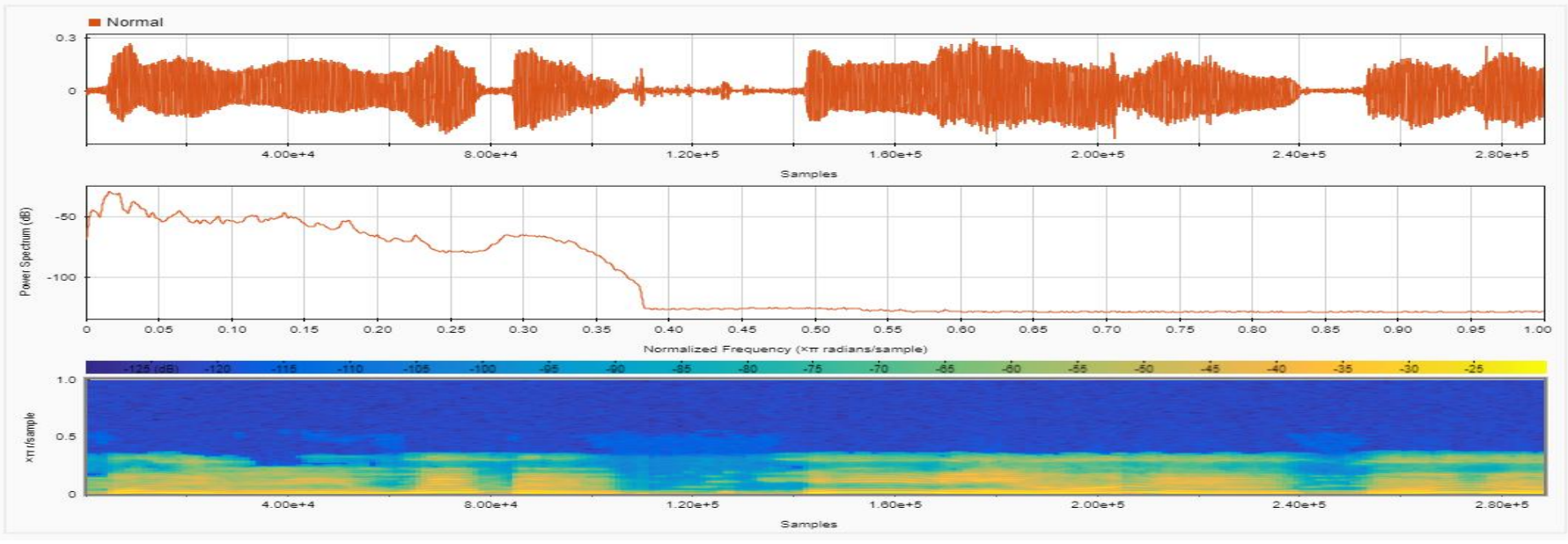

Figure 2: Voice signal of Normal child on time-domain, Spectrum/Frequency, and spectrogram view 

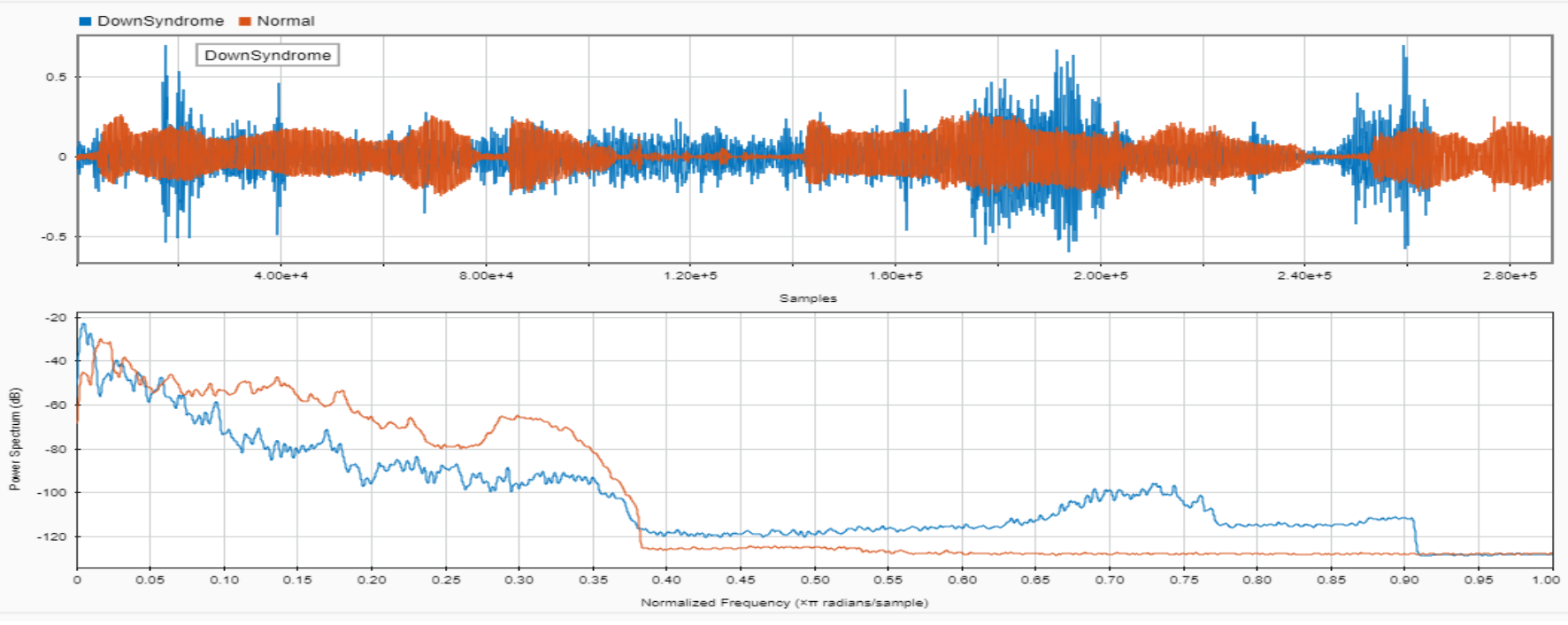

Figure 3: Combination of Down syndrome and Normal child Voice Signal on time-domain and frequency view

Table 1. Comparative Analysis of voice for down syndrome and Normal Child

\begin{tabular}{|c|c|c|}
\hline Parameters & Down Syndrome & Normal \\
\hline Age & 7 years & 7 years \\
\hline Frequency & $44100 \mathrm{~Hz}$ & $48000 \mathrm{~Hz}$ \\
\hline $\begin{array}{c}\text { Normalized } \\
\text { Frequency }\end{array}$ & 0.004914 radius/sample & $\begin{array}{c}0.016356 \\
\text { radius/sample }\end{array}$ \\
\hline Pitch level & $-29.83 \mathrm{db}$ & $-22.7 \mathrm{db}$ \\
\hline Amplitude & 0.001923 meters & 0.000367 meters \\
\hline
\end{tabular}

The level of signal processing of Down syndrome child was higher commenced from zero and over a period of time it improved with a higher level of pitch as it was compared with that of the normal child in figure 2 . In a down syndrome child, the frequency against time plots, it is seen that the frequency level began from the onset of the child, meaning all factors causing down syndrome has been addressed. The second result where both voices placed together (see Figure 3) which shows the compared results for a down syndrome and the normal child. It was observed that the degree of spectrum $(\mathrm{dB})$ set off with a high pitch level, at $-29.83 \mathrm{db}$ for the Down syndrome child, while the normal child is slightly higher than $-22.7 \mathrm{db}$ and it remained relatively stable for a Down syndrome child along the normalized frequency within $0.00491 \mathrm{~Hz}$ and $0.01636 \mathrm{~Hz}$ respectively. Table 1 shows the comparative analysis of the voice for down syndrome and normal child. It was also observed that the results of the investigation show the comparison deduced from the analysis of Down syndrome and of the normal child voice data. The problem of Down syndrome disorder comes with physical appearance: flat, broad head, slanted eyes, pointing upward, small mouth, poor muscle tone during the newborn period, hearing loss and ear infections are frequent. As they grow, children with Down syndrome have shorter than average height and People with Down syndrome have a shortened lifespan. They are also at increased risk of early Alzheimer's disease.

\section{CONCLUSION}

DS is caused by an extra copy of chromosome number 21 inside each of the body's cells. It is a chromosomal accident, not caused by anything the parents have done before or during the pregnancy, and is only very rarely inherited. Early screening tests to look for medical problems associated with Down syndrome are paramount important and it will help in reducing the high risk of Down syndrome disorder. A visit to some medical specialists is recommended as well and including early intervention and special education services. This study has experimental showed through the voice analysis conducted using the signal analyzer tool in MATLAB the difference in the voice of a Down syndrome child and a normal child and the results was compared.

\section{REFERENCES}

[1] Nuallain, S., Flanagan O., Raffat I., Avalos G., \& Dineen B. (2009) The prevalence of Down syndrome in County Galway. Ir Med. 100:329-31.

[2] Carothers A., Hecht C., \& Hook E. (2009) International variation in reported live birth prevalence rates of Down syndrome, adjusted for maternal age. J Med Genet. 36:386-93.

[3] Canfield M., Honein M., Yuskiv N., Xing J, Mai C., \& Collins, J, (2006) National estimates and race/ethnic-specific variation of selected birth defects in the United States. Birth Defects Res A Clin Mol Teratol. 76:747-56.

[4] Murthy S, Malhotra A, Mani S, Shara M, Rowaished E, \& Naveed S, (2007) Incidence of Down syndrome in Dubai. Med Princ Pract. 16:25-8.

[5] Wahab A., Bener A., Teebi A. (2006) The incidence patterns of Down syndrome in Qatar. Clin Genet. 69:360-2.

[6] Mégarbané, A., Ravel A., Mircher C., Sturtz F., Grattau Y, Rethoréet (2009) The $50^{\text {th }}$ anniversary of the discovery of trisomy 21: the past, present, and future of research and treatment of Down syndrome. Genet Med. 11:611-6.

[7] Gardiner K. J. (2010) Molecular basis of pharmacotherapies for cognition in Down syndrome. Trends Pharmacol Sci. 31:66-73.

[8] Prandini P., Deutsch S., Lyle R, Gagnebin M., Delucinge Vivier C., Delorenzi M. (2007) Natural gene-expression variation in 
Down syndrome modulates the outcome of gene-dosage imbalance. Am J Hum Genet. 81:252-63.

[9] Morris, J.K., Wald N.J., \& Watt H.C.( 2019) Fetal loss in Down syndrome pregnancies. Prenat Diagn. 19:142-5.

[10] Glasson, E.J., Sullivan, S.G., Hussain, R., Petterson, B.A., Montgomery, P.D., Bittles, A.H., (2002). The changing survival profile of people with Down's syndrome: implications for genetic counselling. Clin Genet. 62:390-3.

[11] Davis, F. D. (1989). Perceived usefulness, perceived ease of use, and user acceptance of information technology. MIS Quarterly 13 (3): 319-340, doi:10.2307/249008.

[12] Fischer, K. W \& Lamborn, S. D. (1980). Optimal and functional levels in cognitive development: the individual's developmental range. Newsletter of the International society for the study of behavioral development, 2(14): 1-4.

[13] Roizen, N. J, Wolters, C., Nicol, T., Blondis, T.A.(1993). Hearing loss in children with Down syndrome. The Journal of Pediatrics. 123:S9-S12.

[14] Miller, J. \&, Leddy, M. (1999). Verbal fluency, speech intelligibility and communicative effectiveness. In: Miller JF, Leddy M, Leavitt LA, editors. Improving the communication of people with Down syndrome. Baltimore: Paul H. Brookes; pp. 8191.
[15] Laws, G. \& Bishop, DVM(2003). A comparison of language abilities in adolescents with Down syndrome and children with specific language impairment. Journal of Speech, Language, and Hearing Research. ;46:1324-1339.

[16] Peerapol, K. (2009) "Singing Voice Recognition based on Matching of Spectrogram Pattern", Proceedings of International Joint Conference on Neural Networks, Atlanta, Georgia, USA, pp.1595-1599, ISBN: 978-1-4244-3548-7, 2009 IEEE.

[17] Soon, S. J. (2011)," HMM Voice Recognition Algorithm Coding", International Conference on InformationScience and Applications (ICISA), pp. 1-7, ISBN-978-1-4244-9222-0, 2011 IEEE.

[18] Rodger, R.(2009). Voice quality of children and young people with Down's syndrome and its impact on listener judgement. Unpublished PhD thesis. Queen Margaret University; Edinburgh, UK:

[19] Emeeshat, J. S.(2017). Isolated word speech recognition system for children withDown syndrome, M. Sc. Thesisi. Youngstown State University.

[20] Mario, C., David, E., C'esar, G. ,Valentín, C. \& Pastora, M.(2021). Analysis of atypical prosodic patterns in the speech of people with Down syndrome. Biomedical Signal Processing and Control 69 (2021) 102913, 1-11 